Article

\title{
Operations and Aggregation Methods of Single-Valued Linguistic Neutrosophic Interval Linguistic Numbers and Their Decision Making Method
}

\author{
Jun Ye* (iD) and Wenhua Cui \\ Department of Electrical Engineering and Automation, Shaoxing University, 508 Huancheng West Road, \\ Shaoxing 312000, China; wenhuacui@usx.edu.cn \\ * Correspondence: yehjun@aliyun.com; Tel.: +86-575-88327323
}

Received: 17 July 2018; Accepted: 30 July 2018; Published: 1 August 2018

\begin{abstract}
To comprehensively describe uncertain/interval linguistic arguments and confident linguistic arguments in the decision making process by a linguistic form, this study first presents the concept of a single-valued linguistic neutrosophic interval linguistic number (SVLN-ILN), which is comprehensively composed of its uncertain/interval linguistic number (determinate linguistic argument part) and its single-valued linguistic neutrosophic number (confident linguistic argument part), and its basic operations. Then, the score function of SVLN-ILN based on the attitude index and confident degree/level is presented for ranking SVLN-ILNs. After that, SVLN-ILN weighted arithmetic averaging (SVLN-ILNWAA) and SVLN-ILN weighted geometric averaging (SVLN-ILNWGA) operators are proposed to aggregate SVLN-ILN information and their properties are investigated. Further, a multi-attribute decision-making (MADM) method based on the proposed SVLN-ILNWAA or SVLN-ILNWGA operator and the score function is established under consideration of decision makers' preference attitudes (pessimist, moderate, and optimist). Lastly, an actual example is given to show the applicability of the established MADM approach with decision makers' attitudes.
\end{abstract}

Keywords: single-valued linguistic neutrosophic interval linguistic number; score function; weighted aggregation operator; decision making

\section{Introduction}

Multi-attribute decision-making (MADM) explicitly evaluates multiple conflicting attributes in decision making to help people make optimal decisions [1-4]. There usually exists uncertainty and vagueness in MADM problems. In this situation, it may prove difficult for decision makers (DMs) to express their evaluation values of attributes, especially qualitative attributes, by numerical values. Then, the expression of linguistic terms (LTs) is very fit for human thinking and expressing habits. For instance, when the quality of some product is evaluated by LTs, we use LTs "good", "very good", and so on to easily express it. Hence, linguistic decision making methods have been wildly used for MADM problems with linguistic information. Firstly, Zadeh [5] presented the concept of a linguistic variable (LV) for its fuzzy reasoning application. Then, Herrera et al. [6] and Herrera and Herrera-Viedma [7] solved linguistic decision making problems using a linguistic decision analysis. After that, many scholars [8-14] introduced different linguistic aggregation operators for (group) decision making problems. Owing to the uncertainty and vagueness in the linguistic decision environment, uncertain/interval linguistic numbers (ILNs) and various uncertain linguistic aggregation operators have been also presented for uncertain linguistic (group) decision 
making problems [15-20]. Based on a neutrosophic number (i.e., a changeable interval number with indeterminacy), Ye [21] put forward the concept of a neutrosophic linguistic number (NLN), its basic operational laws, and two NLN weighted aggregation operators for multi-attribute group decision making (MAGDM). To represent the hybrid linguistic information of the partial uncertain and partial certain arguments, Ye [22] introduced linguistic cubic numbers (LCNs) and their operations and two weighted aggregation operators for MADM problems with LCN information. To independently depict the truth, falsity, and indeterminacy linguistic arguments in real life for an evaluated object, Fang and Ye [23] presented linguistic neutrosophic numbers (LNNs), their operations, and two weighted aggregation operators for MAGDM in an LNN setting.

In uncertain linguistic MADM problems, it may prove difficult for DMs to give accurate LT values for an attribute from a predefined LT set, but can assign a certain interval linguistic range to it. However, ILN only indicates interval/uncertain LT values of DMs for an attribute, but cannot reflect the confident degree of their judgment. Although Wang et al. [24] proposed the concept of the intuitionistic interval number (IIN) composed of its interval judgment (its uncertain argument) and its intuitionistic fuzzy judgment (its confident judgment) to express the hybrid information of both, IIN cannot express its linguistic argument information in a linguistic evaluation setting. However, how to express the hybrid information of a single-valued LNN and an ILN simultaneously is a difficult problem because there is no research in existing literature. For instance, suppose we give both the ILN $\left[l_{4}, l_{6}\right]$ (the uncertain/interval linguistic argument) and the single-valued LNN $<l_{5}, l_{3}, l_{1}>$ (the confident linguistic judgment) from the given LT set $L=\left\{l_{s} \mid s \in[0,8]\right\}$ regarding an evaluated object. It is obvious that IIN [24] cannot express the hybrid information of both the ILN and the single-valued LNN. To comprehensively describe an uncertain linguistic argument and a confident linguistic judgment in the decision making process, we need the single-valued linguistic neutrosophic ILN (SVLN-ILN), which consists of an ILN and a single-valued LNN (SVLNN), where an SVLNN inflects the confident level/degree of decision makers indicated by the truth, indeterminacy, and falsity LT values corresponding to its ILN judgment for an evaluated object, in order to solve the gap. Therefore, the purposes of this study are as follows: (1) to propose the SVLN-ILN concept for expressing the hybrid information of both the ILN and the single-valued LNN, its operations, and score function with both attitude index and confident level/degree for ranking SVLN-ILNs; (2) to present SVLN-ILN weighted arithmetic averaging (SVLN-ILNWAA) and SVLN-ILN weighted geometric averaging (SVLN-ILNWGA) operators; and (3) to establish an MADM method using the SVLN-ILNWAA or SVLN-ILNWGA operator and the score function to handle MADM problems in SVLN-ILN setting and DMs' attitudes (pessimist, moderate, and optimist).

This study is constructed as per the following structural framework. Section 2 proposes the SVLN-ILN concept composed of ILN and SVLNN, the basic operations of SVLN-ILNs, and the score function of SVLN-ILN for ranking SVLN-ILNs. In Section 3, the SVLN-ILNWAA and SVLN-ILNWGA operators are given to aggregate SVLN-ILNs, and then their properties are discussed. In Section 4, a MADM method with DMs' attitudes is established based on the SVLN-ILNWAA or SVLN-ILNWGA operator and the score function under the SVLN-ILN setting. Section 5 presents an actual example to show the applicability of the proposed MADM method in the SVLN-ILN setting. Lastly, conclusions and future work are indicated in Section 6.

\section{Single-Valued Linguistic Neutrosophic Interval Linguistic Numbers}

Based on the extension of IINs [24], this section presents the concept of SVLN-ILN, which contains the hybrid information of both SVLNN and ILN, the basic operations of SVLN-ILNs, and the score function of SVLN-ILN.

Definition 1. Let a $L T$ set be $L=\left\{l_{s} \mid s \in[0, z]\right\}$, where $z+1$ is an odd number/cardinality. A SVLN-ILN $g$ in $L$ is constructed as $g=\left\langle\left[l_{a}, l_{b}\right] ; l_{T}, l_{I}, l_{F}\right\rangle$, where $\left[l_{a}, l_{b}\right]$ is the ILN part of $g$ and $l_{a}$ and $l_{b}$ are linguistic lower 
and upper bounds of $l_{S}$ for $l_{a} \leq l_{s} \leq l_{b}$ and $l_{S} \in L$, and then $<l_{T}, l_{I}, l_{F}>$ is the SVLNN part of $g$. Here, the truth linguistic function $T_{g}\left(l_{s}\right)$ of $g$ can be defined as

$$
T_{g}\left(l_{s}\right)= \begin{cases}l_{T}, & l_{a} \leq l_{s} \leq l_{b} \\ l_{0}, & \text { otherwise }\end{cases}
$$

The indeterminacy linguistic function $I_{g}\left(l_{s}\right)$ of $g$ can be defined as

$$
I_{g}\left(l_{s}\right)= \begin{cases}l_{I}, & l_{a} \leq l_{s} \leq l_{b} \\ l_{z}, & \text { otherwise }\end{cases}
$$

The falsity linguistic function $F_{g}\left(l_{s}\right)$ of $g$ can be defined as

$$
F_{g}\left(l_{s}\right)= \begin{cases}l_{F}, & l_{a} \leq l_{s} \leq l_{b} \\ l_{z}, & \text { otherwise }\end{cases}
$$

where $l_{0} \leq l_{T} \leq l_{z}, l_{0} \leq l_{I} \leq l_{z}$, and $l_{0} \leq l_{F} \leq l_{z}$.

For instance, $g=\left\langle\left[l_{4}, l_{6}\right] ; l_{5}, l_{2}, l_{3}>\right.$ is an SVLN-ILN, where $\left[l_{4}, l_{6}\right]$ is the ILN part of $g$, and then $<l_{5}, l_{2}, l_{3}>$ is the SVLNN part depicted by the truth linguistic value $l_{5}$, the indeterminacy linguistic value $l_{2}$, and the falsity linguistic value $l_{3}$, independently. In a decision making problem, the SVLN-ILN indicates both DMs' interval linguistic judgment (uncertain linguistic judgment) and confident linguistic judgment for an evaluated object.

To express the semantics conveniently, we adopt a linguistic transformation/scale function $f\left(l_{s}\right)=s$ for $s \in[0, z]$, which produces the mapping $f: l_{s} \rightarrow s$, i.e., the mapping from a LT in $L=\left\{l_{s} \mid s \in[0, z]\right\}$ to a numerical value.

Definition 2. Suppose $g_{1}=\left\langle\left[l_{a_{1}}, l_{b_{1}}\right] ; l_{T_{1}}, l_{I_{1}}, l_{F_{1}}\right\rangle$ and $g_{2}=\left\langle\left[l_{a_{2}}, l_{b_{2}}\right] ; l_{T_{2}}, l_{I_{2}}, l_{F_{2}}\right\rangle$ are two SVLN-ILNs in L. If their arguments/expected values are $m_{1}=\left[f\left(l_{a_{1}}\right)+f\left(l_{b_{1}}\right)\right] / 2=\left(a_{1}+b_{1}\right) / 2$ and $m_{2}=\left[f\left(l_{a_{2}}\right)+\right.$ $\left.f\left(l_{b_{2}}\right)\right] / 2=\left(a_{2}+b_{2}\right) / 2$, and a positive scalar is $p>0$, their basic operations can be defined below:

$$
\begin{aligned}
& g_{1} \oplus g_{2}=\left\langle\left[l_{a_{1}+a_{2}-\frac{a_{1} \cdot a_{2}}{z}}, l_{b_{1}+b_{2}-\frac{b_{1} \cdot b_{2}}{z}}^{z}\right] ; l_{\frac{m_{1} T_{1}+m_{2} T_{2}}{m_{1}+m_{2}}}, \frac{l_{m_{1} I_{1}+m_{2} I_{2}}}{m_{1}+m_{2}}, \frac{l_{m_{1} F_{1}+m_{2} F_{2}}}{m_{1}+m_{2}}\right\rangle
\end{aligned}
$$

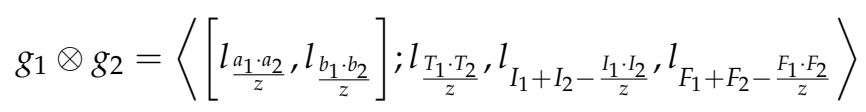

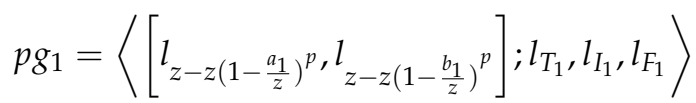

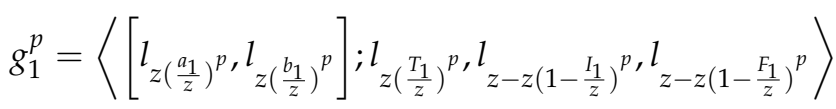

Clearly, the above calculated results are still SVLN-ILNs.

Example 1. Suppose $g_{1}=<\left[l_{4}, l_{6}\right] ; l_{5}, l_{2}, l_{3}>$ and $g_{2}=<\left[l_{2}, l_{6}\right] ; l_{6}, l_{1}, l_{2}>$ are two SVLN-ILNs in the LT set $L=\left\{l_{0}, l_{1}, \ldots, l_{8}\right\}$ for $z=8$ and $p=2$. Then, their arguments are $m_{1}=\left[f\left(l_{4}\right)+f\left(l_{6}\right)\right] / 2=(4+6) / 2=5$ and $m_{2}=\left[f\left(l_{2}\right)+f\left(l_{6}\right)\right] / 2=(2+6) / 2=4$, respectively.

Thus, using Equations (1)-(4), the operational results are yielded as follows: 


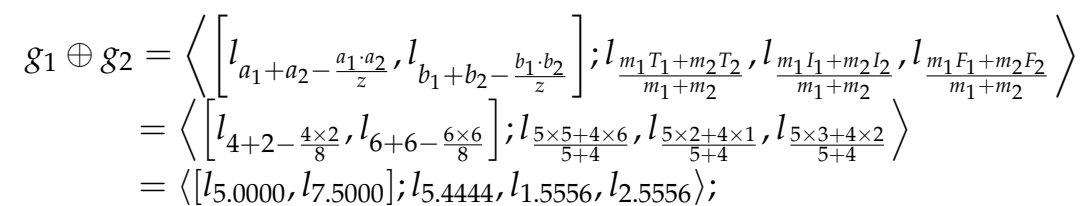

$g_{1} \otimes g_{2}=\left\langle\left[l_{\frac{a_{1} \cdot a_{2}}{z}}, l_{\frac{b_{1} \cdot b_{2}}{z}}\right] ; l_{\frac{T_{1} \cdot T_{2}}{z}}, l_{I_{1}+I_{2}-\frac{I_{1} \cdot I_{2}}{z}}, l_{F_{1}+F_{2}-\frac{F_{1} \cdot F_{2}}{z}}\right\rangle$

$=\left\langle\left[l_{\frac{4 \times 2}{8}}, l_{\frac{6 \times 6}{8}}\right] ; l_{\frac{5 \times 6}{8}}, l_{2+1-\frac{2 \times 1}{8}}, l_{3+2-\frac{3 \times 2}{8}}\right\rangle=\left\langle\left[l_{1.0000}, l_{4.5000}\right] ; l_{3.7500}, l_{2.7500}, l_{4.2500}\right\rangle$;

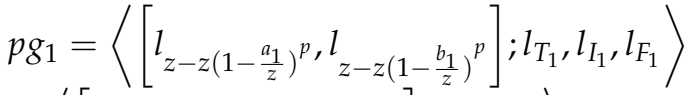

$=\left\langle\left[l_{8-8\left(1-\frac{4}{8}\right)^{2}}, l_{8-8\left(1-\frac{6}{8}\right)^{2}}\right] ; l_{5}, l_{2}, l_{3}\right\rangle=\left\langle\left[l_{6.0000}, l_{7.5000}\right] ; l_{5}, l_{2}, l_{3}\right\rangle$;

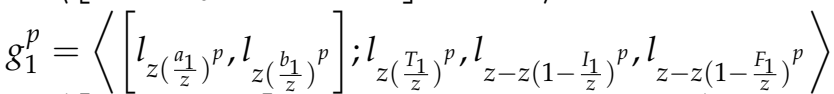

$=\left\langle\left[l_{8\left(\frac{4}{8}\right)^{2}}, l_{8\left(\frac{6}{8}\right)^{2}}\right] ; l_{8\left(\frac{5}{8}\right)^{2}}, l_{8-8\left(1-\frac{2}{8}\right)^{2}}, l_{8-8\left(1-\frac{3}{8}\right)^{2}}\right\rangle=\left\langle\left[l_{2.0000}, l_{4.5000}\right] ; l_{3.1250}, l_{3.5000}, l_{4.8750}\right\rangle$.

For comparison between SVLN-ILNs, both ILN and SVLNN in an SVLN-ILN $g=\left\langle\left[l_{a}, l_{b}\right] ; l_{T}, l_{I}, l_{F}>\right.$ should be considered as the score function containing both the attitude index of ILN and the score value of SVLNN (the confidence level/degree) regarding DMs in the decision making process.

Based on the extension of attitude index for an interval number [25], the attitude index of an ILN $\left[l_{a}, l_{b}\right]$ is defined as follows:

$$
A=\frac{f\left(l_{a}\right)+f\left(l_{b}\right)}{2 z}+(2 \alpha-1) \frac{f\left(l_{b}\right)-f\left(l_{a}\right)}{2 z}=\frac{a+b}{2 z}+(2 \alpha-1) \frac{b-a}{2 z}
$$

where $\alpha \in[0,1]$ is the attitude coefficient.

Then, the score value of SVLNN is given as follows:

$$
S=\frac{2 z+f\left(l_{T}\right)-f\left(l_{I}\right)-f\left(l_{F}\right)}{3 z}=\frac{2 z+T-I-F}{3 z}
$$

Thus, the score function of a SVLN-ILN can be given by the definition below.

Definition 3. Based on the combination of both the attitude index of ILN and the score value of SVLN (the confidence level) for a SVLN-ILN $g=\left\langle\left[l_{a}, l_{b}\right] ; l_{T}, l_{I}, l_{F}\right\rangle$, the new score function of a SVLN-ILN can be given as

$$
Y(g)=A \times S=\left(\frac{a+b}{2 z}+(2 \alpha-1) \frac{b-a}{2 z}\right) \times\left(\frac{2 z+T-I-F}{3 z}\right) \text { for } Y(g)[0,1]
$$

In the score function (7), both the attitude coefficient $\alpha$ and the confident level/score value of $S$ can indicate the pessimistic/moderate/optimistic degree and confident degree of DMs. On the one hand, when DM believes that the linguistic evaluation value of an attribute is in an ILN $\left[l_{a}, l_{b}\right]$, his/her linguistic evaluation value tends to the lower bound $l_{a}$ for a pessimistic DM, conversely, his/her linguistic evaluation value tends to the upper bound $l_{b}$ for an optimistic DM, while his/her linguistic evaluation value tends to the moderate value $\left[f\left(l_{a}\right)+f\left(l_{b}\right)\right] / 2$ for a moderate DM. Obviously, the DM's attitude is increasingly more optimistic with increasing $\alpha$ from 0 to 1 . Especially when $\alpha=0,0.5$, and 1 , the three attitude coefficients reflect the pessimistic, moderate, and optimistic attitudes of DM, respectively. On the other hand, the score value of $S \in[0,1]$ also indicates the confident level/degree of DM. Then, the DM's confident degree is increasingly more high with increasing $S$ from 0 to 1 . Especially $S=1$ for $f\left(l_{T}\right)=\mathrm{z}, f\left(l_{I}\right)=0$, and $f\left(l_{F}\right)=0$ in SVLNN is quite confident; while $S=0$ for $f\left(l_{T}\right)=0$, $f\left(l_{I}\right)=\mathrm{z}$, and $f\left(l_{F}\right)=\mathrm{z}$ in SVLNN is quite unconfident. 
Example 2. Suppose $g=\left\langle\left[l_{6}, l_{7}\right] ; l_{6}, l_{2}, l_{3}\right\rangle$ is the SVLN-ILN in the LT set $L=\left\{l_{s} \mid s \in[0,8]\right\}$ for $z=8$. Then, the pessimistic, moderate, and optimistic attitudes of DM are given by $\alpha=0,0.5,1$, respectively.

Thus, by Equation (7), we calculate the score value of the SVLN-ILN below:

$$
\begin{aligned}
& Y(g)=\left(\frac{a+b}{2 z}+(2 \alpha-1) \frac{b-a}{2 z}\right)\left(\frac{2 z+T-I-F}{3 z}\right) \\
& =\left(\frac{6+8}{2 \times 8}+(2 \alpha-1) \frac{8-6}{2 \times 8}\right)\left(\frac{2 \times 8+6-2-3}{3 \times 8}\right)=\left\{\begin{array}{l}
0.5313 \text { for } \alpha=0, \\
0.6198 \text { for } \alpha=0.5, \\
0.7083 \text { for } \alpha=1
\end{array}\right.
\end{aligned}
$$

Clearly, the score values of SVLN-ILN can be changed with the pessimistic, moderate, and optimistic attitudes of DM (i.e., $\alpha=0,0.5$, and 1 , respectively).

Definition 4. Suppose $g_{1}=\left\langle\left[l_{a_{1}}, l_{b_{1}}\right] ; l_{T_{1}}, l_{I_{1}}, l_{F_{1}}\right\rangle$ and $g_{2}=\left\langle\left[l_{a_{2}}, l_{b_{2}}\right] ; l_{T_{2}}, l_{I_{2}}, l_{F_{2}}\right\rangle$ are two SVLN-ILNs in L, we give the following ranking relations:

(i) If $Y\left(g_{1}\right)>Y\left(g_{2}\right)$, then $g_{1} \succ g_{2}$;

(ii) If $Y\left(g_{1}\right)<Y\left(g_{2}\right)$, then $g_{1} \prec g_{2}$;

(iii) If $Y\left(g_{1}\right)=Y\left(g_{2}\right)$, then $g_{1}=g_{2}$.

Example 3. If $g_{1}=\left\langle\left[l_{5}, l_{7}\right] ; l_{7}, l_{2}, l_{1}>\right.$ and $g_{2}=\left\langle\left[l_{6}, l_{8}\right] ; l_{5}, l_{3}, l_{4}\right\rangle$ are two SVLN-ILNs in the LT set $L=\left\{l_{s} \mid \mathrm{s}\right.$ $\in[0,8]\}$ for $z=8$, they are ranked by DM with the moderate attitude $\alpha=0.5$.

By applying Equation (7), there exists $Y\left(g_{1}\right)=0.6250>Y\left(g_{2}\right)=0.5104$, then $g_{1} \succ g_{2}$.

\section{Weighted Aggregation Operators of SVLN-ILNs}

\subsection{SVLN-ILNWAA Operator}

Definition 5. Suppose $g_{k}=\left\langle\left[l_{a_{k}}, l_{b_{k}}\right] ; l_{T_{k}}, l_{I_{k}}, l_{F_{k}}\right\rangle(k=1,2, \ldots, n)$ is a group of SVLN-ILNs in L. Then, the SVLN-ILNWAA operator can be given as follows:

$$
S V L N-I L N W A A\left(g_{1}, g_{2}, \ldots, g_{n}\right)=\sum_{k=1}^{n} \omega_{k} g_{k}
$$

where $\omega_{k} \in[0,1]$ is the weight of $g_{k}(k=1,2, \ldots, n)$ and $\sum_{k=1}^{n} \omega_{k}=1$.

Thus, the following theorem can be given based on Equations (1), (3), and (8).

Theorem 1. Suppose $g_{k}=\left\langle\left[l_{a_{k}}, l_{b_{k}}\right] ; l_{T_{k}}, l_{I_{k}}, l_{F_{k}}\right\rangle(k=1,2, \ldots, n)$ is a group of SVLN-ILNs in L. Thus, the aggregation result regarding Equation (8) is also a SVLN-ILN, which is yielded by the aggregation form:

$$
\begin{aligned}
& S V L N-I L N W A A\left(g_{1}, g_{2}, \ldots, g_{n}\right)=\sum_{k=1}^{n} \omega_{k} g_{k}
\end{aligned}
$$

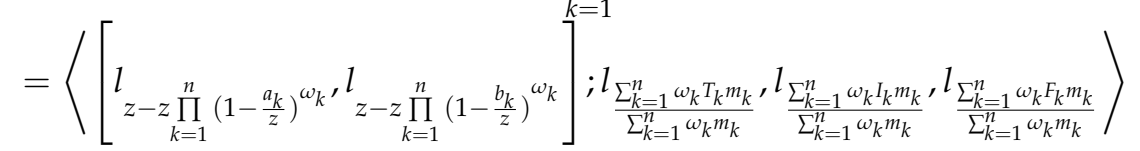

where $m_{k}=\left(a_{k}+b_{k}\right) / 2$ for $a_{k}, b_{k} \in[0, z]$ and $k=1,2, \ldots, n$.

Then, Theorem 1 can be proofed by the mathematical induction. 


\section{Proof:}

If $k=2$, by Equation (3), we have

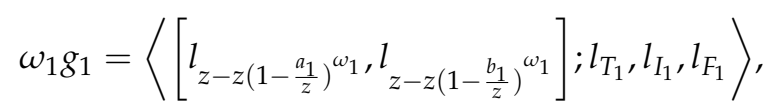

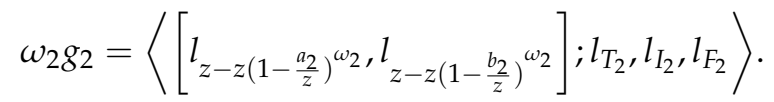

By Equation (1), there exists the following result:

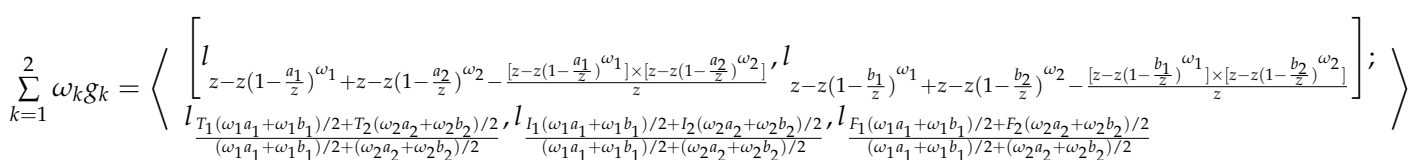

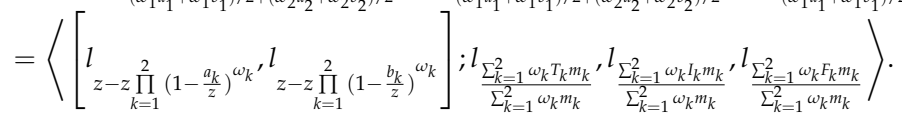

If $k=n$, Equation (9) exists as the following form:

$$
\begin{aligned}
& \text { SVLN }-I L N W A A\left(g_{1}, g_{2}, \ldots, g_{n}\right)=\sum_{k=1}^{n} \omega_{k} g_{k} \\
& =\left\langle\left[l l_{z-z \prod_{k=1}^{n}\left(1-\frac{a_{k}}{z}\right)^{\omega_{k}}, l} l_{z-z \prod_{k=1}^{n}\left(1-\frac{b_{k}}{z}\right)}^{\omega_{k}}\right] ; l_{\frac{\sum_{k=1}^{n} \omega_{k} T_{k} m_{k}}{\sum_{k=1}^{n} \omega_{k} m_{k}}}, l_{\frac{\sum_{k=1}^{n} \omega_{k} I_{k} m_{k}}{\sum_{k=1}^{n} \omega_{k} m_{k}}} l_{\frac{\sum_{k=1}^{n} \omega_{k} F_{k} m_{k}}{\sum_{k=1}^{n} \omega_{k} m_{k}}}\right\rangle .
\end{aligned}
$$

Thus, if $k=n+1$, by Equations (1), (3), and (10), we yield the result:

$$
\begin{aligned}
& \operatorname{SVLN}-\operatorname{ILNWAA}\left(g_{1}, g_{2}, \ldots, g_{n}, g_{k+1}\right)=\sum_{k=1}^{n+1} \omega_{k} g_{k}=\sum_{k=1}^{n} \omega_{k} g_{k} \oplus \omega_{n+1} g_{n+1}
\end{aligned}
$$

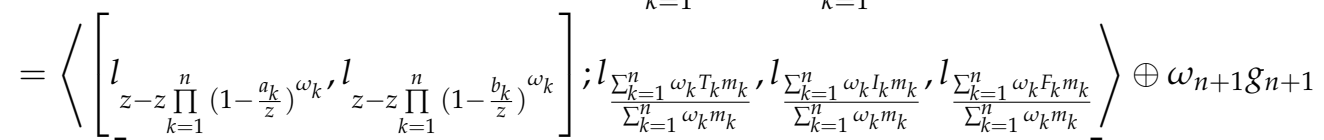

$$
\begin{aligned}
& =\left\langle\left[\begin{array}{c}
l \\
z-z \prod_{k=1}^{n}\left(1-\frac{a_{k}}{z}\right)^{\omega_{k}}+z-z\left(1-\frac{a_{n+1}}{z}\right)^{\omega_{n+1}}-\frac{\left[z-z \prod_{k=1}^{n}\left(1-\frac{a_{k}}{z}\right)^{\omega_{k}}\right] \times\left[z-z\left(1-\frac{a_{n+1}}{z}\right)^{\omega_{n+1}}\right]^{\prime}}{z} \\
z-z \prod_{k=1}^{n}\left(1-\frac{b_{k}}{z}\right)^{\omega_{k}}+z-z\left(1-\frac{b_{n+1}}{z}\right)^{\omega_{n+1}}-\frac{\left[z-z \prod_{k=1}^{n}\left(1-\frac{b_{k}}{z}\right)^{\omega_{k}}\right] \times\left[z-z\left(1-\frac{b_{n+1}}{z}\right)^{\omega_{n+1}}\right]}{z}
\end{array}\right] ;\right\rangle \\
& \frac{l_{\sum_{k=1}^{n} \omega_{k} T_{k} m_{k}+\omega_{n+1} T_{n+1} m_{n+1}}^{\sum_{k=1}^{n} \omega_{k} m_{k}+\omega_{n+1} m_{n+1}}}{\frac{\sum_{k=1}^{n} \omega_{k} I_{k} m_{k}+\omega_{n+1} I_{n+1} m_{n+1}}{\sum_{k=1}^{n} \omega_{k} m_{k}+\omega_{n+1} m_{n+1}}}, \frac{l_{\sum_{k=1}^{n} \omega_{k} F_{k} m_{k}+\omega_{n+1} F_{n+1} m_{n+1}}}{\sum_{k=1}^{n} \omega_{k} m_{k}+\omega_{n+1} m_{n+1}} \\
& =\left\langle\left[\begin{array}{l}
l \\
z-z \prod_{k=1}^{n+1}\left(1-\frac{a_{k}}{z}\right)^{\omega_{k}}, l \\
z-z \prod_{k=1}^{n+1}\left(1-\frac{b_{k}}{z}\right)
\end{array}\right)^{\omega_{k}} ;_{\frac{\sum_{k=1}^{n+1} \omega_{k} T_{k} m_{k}}{\sum_{k=1}^{n+1} \omega_{k} m_{k}}}, l_{\frac{\sum_{k=1}^{n+1} \omega_{k} I_{k} m_{k}}{\sum_{k=1}^{n+1} \omega_{k} m_{k}}} l_{\frac{\sum_{k=1}^{n+1} \omega_{k} F_{k} m_{k}}{\sum_{k=1}^{n+1} \omega_{k} m_{k}}}\right\rangle .
\end{aligned}
$$

Corresponding to the above results, Equation (9) can hold for any $k$. This proof is completed.

To illustrate the operational process of the SVLN-ILNWAA operator, we give the following example.

Example 4. Suppose $g_{1}=\left\langle\left[l_{5}, l_{6}\right] ; l_{5}, l_{2}, l_{1}\right\rangle, g_{2}=\left\langle\left[l_{5}, l_{7}\right] ; l_{6}, l_{3}, l_{1}\right\rangle$, and $\left.g_{3}=<\left[l_{6}, l_{7}\right] ; l_{7}, l_{3}, l_{3}\right\rangle$ are three SVLN-ILNs in the $L T$ set $L=\left\{l_{S} \mid s \in[0,8]\right\}$ for $z=8$, then their weigh vector is $\omega=(0.32,0.25,0.43)$.

Thus, there are $m_{1}=(5+6) / 2=5.5, m_{2}=(5+7) / 2=6$, and $m_{3}=(6+7) / 2=6.5$. 
Using Equation (9), their operational result of the SVLN-ILNWAA operator is given below:

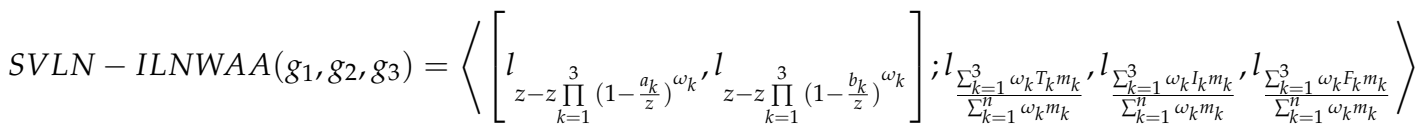

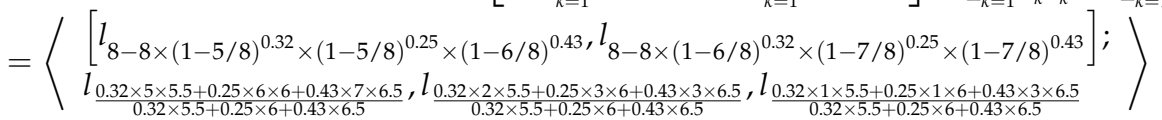

$$
\begin{aligned}
& =\left\langle\left[l_{5.4800}, l_{6.7517}\right] ; l_{6.1709}, l_{2.7093}, l_{1.9232}\right\rangle \text {. }
\end{aligned}
$$

Obviously, their operational result of the SVLN-ILNWAA operator is also an SVLN-ILN and all the LT values in it still belong to $L$.

Theorem 2. Suppose $g_{k}=\left\langle\left[l_{a_{k}}, l_{b_{k}}\right] ; l_{T_{k}}, l_{I_{k}}, l_{F_{k}}\right\rangle(k=1,2, \ldots, n)$ is a group of SVLN-ILNs in L. Thus, the SVLN-ILNWAA operator implies these properties:

(1) Idempotency: Set $g_{k}(k=1,2, \ldots, n)$ as a group of SVLN-ILNs in $L$. If $g_{k}=g$ for $k=1,2, \ldots, n$, then there exists $S V L N-I L N W A A\left(g_{1}, g_{2}, \cdots, g_{n}\right)=g$.

(2) Boundedness: Suppose $g_{k}(k=1,2, \ldots, n)$ is a group of SVLN-ILNs in $L$. Let the minimum SVLN-ILN be $g^{-}=\left\langle\left[\min _{k} f\left(l_{a_{k}}\right), \min _{k}\left(l_{b_{k}}\right)\right] ; \min _{k} f\left(l_{T_{k}}\right), \max _{k} f\left(l_{I_{k}}\right), \max _{k} f\left(l_{F_{k}}\right)\right\rangle$ and the maximum SVLN-ILN be $g^{+}=\left\langle\left[\max _{k} f\left(l_{a_{k}}\right), \max _{k} f\left(l_{b_{k}}\right)\right], \max _{k}\left(l_{T_{k}}\right), \min _{k}\left(l_{I_{k}}\right), \min _{k}\left(l_{F_{k}}\right)\right\rangle$. Then, $g^{-} \leq S V L N-I L N W A A\left(g_{1}, g_{2}, \cdots, g_{n}\right) \leq g^{+}$can hold.

(3) Monotonicity: Suppose $g_{k}(k=1,2, \ldots, n)$ is a group of SVLN-ILNs in $L$. If $g_{k} \leq g_{k}^{*}$ for $k=1,2, \ldots, n$, then $\operatorname{SVLN}-\operatorname{ILNWAA}\left(g_{1}, g_{2}, \cdots, g_{n}\right) \leq \operatorname{SVLN}-\operatorname{ILNWAA}\left(g_{1}^{*}, g_{2}^{*}, \cdots, g_{n}^{*}\right)$ can hold.

\section{Proof:}

(1) Because $g_{k}=g$ for $k=1,2, \ldots, n$, there is the following result:

$$
\begin{aligned}
& \operatorname{SVLN}-\operatorname{ILNWAA}\left(g_{1}, g_{2}, \ldots, g_{n}\right)=\sum_{k=1}^{n} \omega_{k} g_{k}
\end{aligned}
$$

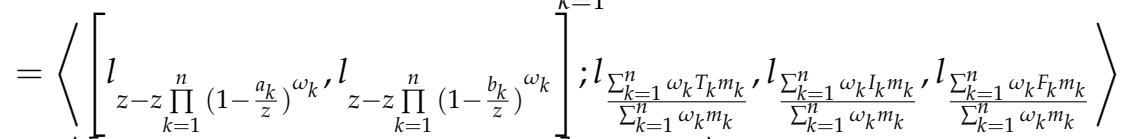

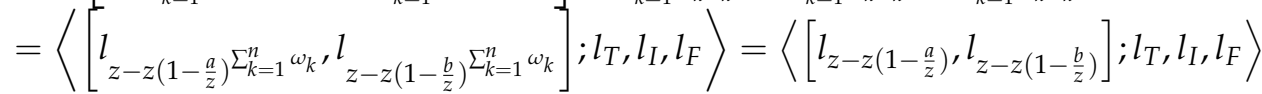

$$
\begin{aligned}
& =\left\langle\left[l_{a}, l_{b}\right] ; l_{T}, l_{I}, l_{F}\right\rangle=g \text {. }
\end{aligned}
$$

(2) Because $g^{-}$is the minimum SVLN-ILN and $g^{+}$is the maximum SVLN-ILN, $g^{-} \leq g_{k} \leq g^{+}$holds. Hence, $\sum_{k=1}^{n} \omega_{j} g^{-} \leq \sum_{k=1}^{n} \omega_{k} g_{k} \leq \sum_{k=1}^{n} \omega_{k} g^{+}$can hold. There exists $g^{-} \leq \sum_{k=1}^{n} \omega_{k} g_{k} \leq g^{+}$according to the property (1), that is, $g^{-} \leq S V L N-I L N W A A\left(g_{1}, g_{2}, \cdots, g_{n}\right) \leq g^{+}$.

(3) For $g_{k} \leq g_{k}^{*}(k=1,2, \ldots, n), \sum_{k=1}^{n} \omega_{k} g_{k} \leq \sum_{k=1}^{n} \omega_{k} g_{k}^{*}$ can hold, that is, SVLN $\operatorname{ILNWAA}\left(g_{1}, g_{2}, \cdots, g_{n}\right) \leq \operatorname{SVLN}-\operatorname{ILNWAA}\left(g_{1}^{*}, g_{2}^{*}, \cdots, g_{n}^{*}\right)$.

Thus, the proof of these properties is finished.

Especially when $\omega_{k}=1 / n$ for $k=1,2, \ldots, n$, the SVLN-ILNWAA operator reduces to the SVLN-ILN arithmetic average operator. 


\subsection{SVLN-ILNWGA Operator}

Definition 6. Suppose $g_{k}=\left\langle\left[l_{a_{k}}, l_{b_{k}}\right] ; l_{T_{k}}, l_{I_{k}}, l_{F_{k}}\right\rangle(k=1,2, \ldots, n)$ is a group of SVLN-ILNs in L. Then, we give the following definition of the SVLN-ILNWGA operator:

$$
S V L N-\operatorname{ILNWGA}\left(g_{1}, g_{2}, \cdots, g_{n}\right)=\prod_{k=1}^{n} g_{k}^{\omega_{k}}
$$

where $\omega_{k} \in[0,1]$ is the weight of $g_{k}(k=1,2, \ldots, n)$ and $\sum_{k=1}^{n} \omega_{k}=1$.

Then, we can give the following theorem based on Equations (2), (4), and (11).

Theorem 3. Suppose $g_{k}=\left\langle\left[l_{a_{k}}, l_{b_{k}}\right] ; l_{T_{k}}, l_{I_{k}}, l_{F_{k}}\right\rangle(k=1,2, \ldots, n)$ is a group of SVLN-ILNs in L. Thus, the aggregation result of Equation (11) is also an SVLN-ILN, which is obtained by the aggregation form:

$$
\begin{aligned}
& S V L N-I L N W G A\left(g_{1}, g_{2}, \cdots, g_{n}\right)=\prod_{k=1}^{n} g_{k}^{\omega_{k}} \\
& =\left\langle\left[l_{z \prod_{k=1}^{n}\left(\frac{a_{k}}{z}\right){ }^{\omega_{k}}, l_{z} \prod_{k=1}^{n}\left(\frac{b_{k}}{z}\right)}^{\omega_{k}}\right] ; l_{z \prod_{k=1}^{n}\left(\frac{T_{k}}{z}\right)}{ }^{\omega_{k}}, l_{z-z \prod_{k=1}^{n}\left(1-\frac{I_{k}}{z}\right)}^{\omega_{k}}, l_{z-z \prod_{k=1}^{n}\left(1-\frac{F_{k}}{z}\right)} \omega_{k}\right\rangle .
\end{aligned}
$$

Similar to the proof of Theorem 1, Theorem 3 can also be proved below.

\section{Proof:}

If $k=2$, by Equation (4) we get

$$
\begin{aligned}
& g_{1}^{\omega_{1}}=\left\langle\left[l_{z\left(\frac{a_{1}}{z}\right)}{ }^{\omega_{1}}, l_{z\left(\frac{b_{1}}{z}\right)}{ }^{\omega_{1}}\right] ; l_{z\left(\frac{T_{1}}{z}\right)}{ }^{\omega_{1}}, l_{z-z\left(1-\frac{I_{1}}{z}\right)}{ }^{\omega_{1}}, l_{z-z\left(1-\frac{F_{1}}{z}\right)}{ }^{\omega_{1}}\right\rangle, \\
& g_{2}^{\omega_{2}}=\left\langle\left[l_{z\left(\frac{a_{2}}{z}\right)} \omega_{2}, l_{z\left(\frac{b_{2}}{z}\right)} \omega_{2}\right] ; l_{z\left(\frac{T_{2}}{z}\right)} \omega_{2}, l_{z-z\left(1-\frac{I_{2}}{z}\right)} \omega_{2}, l_{z-z\left(1-\frac{F_{2}}{z}\right)} \omega_{2}\right\rangle .
\end{aligned}
$$

Using Equation (2), there exists the following result:

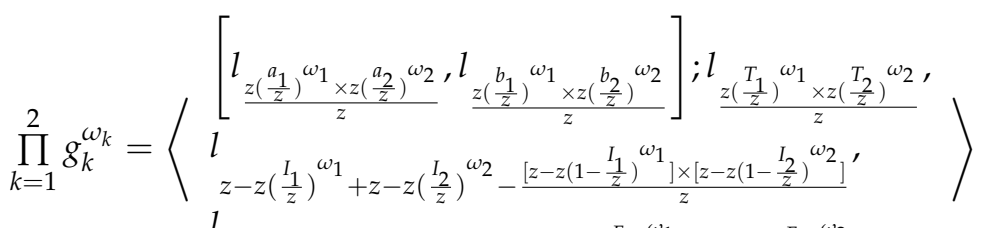

$$
\begin{aligned}
& l=z\left(\frac{F_{1}}{z}\right)^{\omega_{1}}+z-z\left(\frac{F_{2}}{z}\right)^{\omega_{2}}-\frac{\left[z-z\left(1-\frac{F_{1}}{z}\right)^{\omega_{1}}\right] \times\left[z-z\left(1-\frac{F_{2}}{z}\right)^{\left.\omega_{2}\right]}\right.}{z}
\end{aligned}
$$

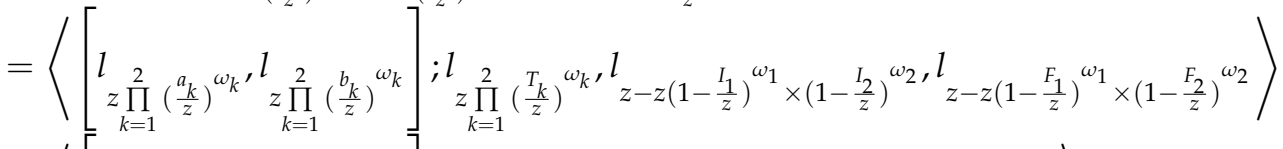

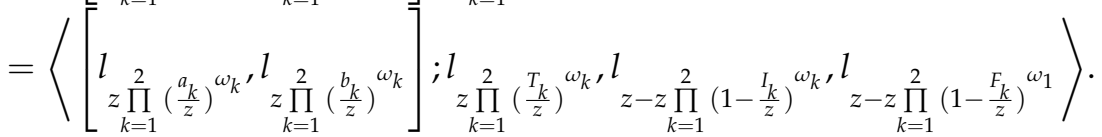

If $k=n$, Equation (12) exists as the following result:

$$
\begin{aligned}
& S V L N-I L N W G A\left(g_{1}, g_{2}, \cdots, g_{n}\right)=\prod_{k=1}^{n} g_{k}^{\omega_{k}} \\
& =\left\langle\left[l z \prod_{k=1}^{n}\left(\frac{a_{k}}{z}\right) \omega_{k}, l z \prod_{k=1}^{n}\left(\frac{b_{k}}{z}\right) \omega_{k}\right] ; l z \prod_{k=1}^{n}\left(\frac{T_{k}}{z}\right)^{\omega_{k}}, l z-z \prod_{k=1}^{n}\left(1-\frac{I_{k}}{z}\right)^{\omega_{k}}, l z-z \prod_{k=1}^{n}\left(1-\frac{F_{k}}{z}\right) \omega_{k}\right\rangle .
\end{aligned}
$$


Thus if $k=n+1$, by Equations (2), (4), and (13), we yield the following result:

$$
\begin{aligned}
& S V L N-I L N W G A\left(g_{1}, g_{2}, \ldots, g_{n}, g_{n+1}\right)=\prod_{k=1}^{n+1} g_{k}^{\omega_{k}}=\prod_{k=1}^{n} g_{k}^{\omega_{k}} \otimes g_{n+1}^{\omega_{n+1}}
\end{aligned}
$$

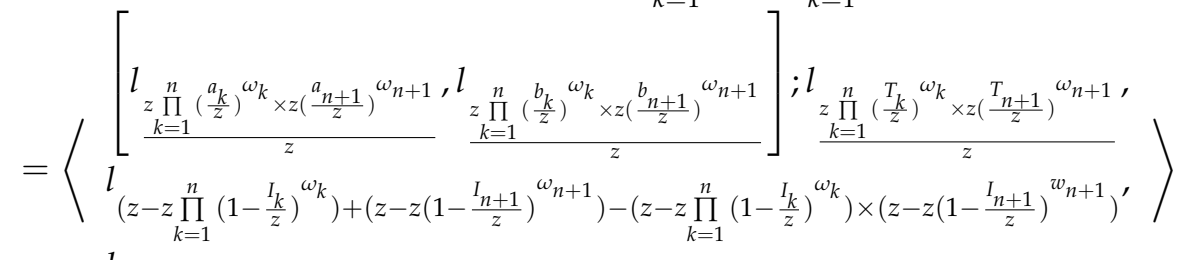

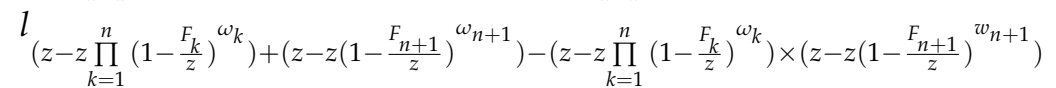

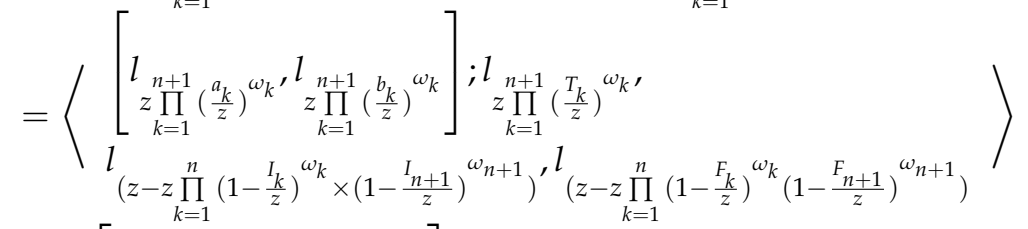

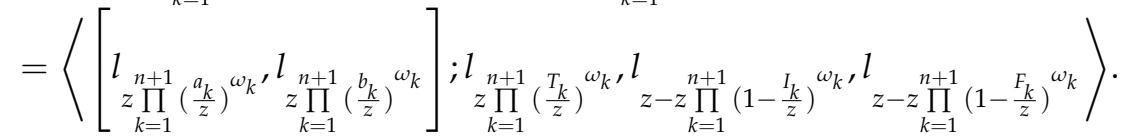

Based on the above results, Equation (12) exists for any $k$. This proof is finished.

Example 5. Consider Example 4 to calculate the aggregation result of the SVLN-ILNWGA operator.

By Equation (12), the calculation process is shown as follows:

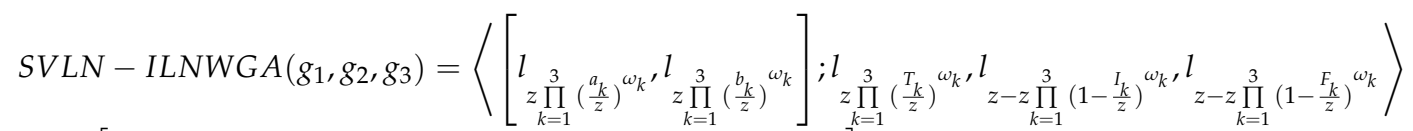

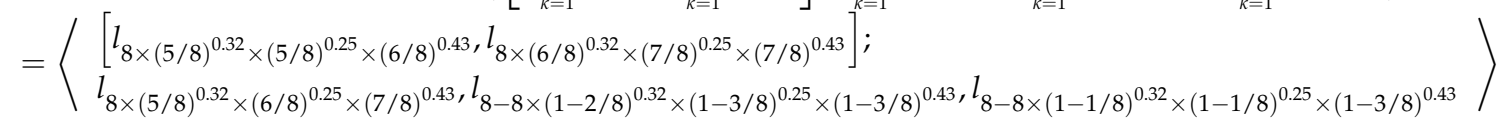

$$
\begin{aligned}
& =\left\langle\left[l_{5.4078}, l_{6.6631}\right] ; l_{6.0478}, l_{2.6996}, l_{1.9429}\right\rangle \text {. }
\end{aligned}
$$

Obviously, their operational result of the SVLN-ILNWGA operator is also a SVLN-ILN and all the LT values in it still belong to $L$.

Theorem 4. Suppose $g_{k}=\left\langle\left[l_{a_{k}}, l_{b_{k}}\right] ; l_{T_{k}}, l_{I_{k}}, l_{F_{k}}\right\rangle(k=1,2, \ldots, n)$ is a group of SVLN-ILNs in L. Thus, the SVLN-ILNWGA operator indicates these properties:

(1) Idempotency: Suppose $g_{k}(k=1,2, \ldots, n)$ is a group of SVLN-ILNs in $L$. If $g_{k}=g$ for $k=1,2, \ldots$, $n$, then there exists $S V L N-I L N W G A\left(g_{1}, g_{2}, \cdots, g_{n}\right)=g$.

(2) Boundedness: Suppose $g_{k}(k=1,2, \ldots, n)$ is a group of SVLN-ILNs in $L$. Let the minimum SVLN-ILN be $g^{-}=\left\langle\left[\min _{k} f\left(l_{a_{k}}\right), \min _{k}\left(l_{b_{k}}\right)\right] ; \min _{k} f\left(l_{T_{k}}\right), \max _{k} f\left(l_{I_{k}}\right), \max _{k} f\left(l_{F_{k}}\right)\right\rangle$ and the maximum SVLN-ILN be $g^{+}=\left\langle\left[\max _{k} f\left(l_{a_{k}}\right), \max _{k} f\left(l_{b_{k}}\right)\right], \max _{k}\left(l_{T_{k}}\right), \min _{k}\left(l_{I_{k}}\right), \min _{k}\left(l_{F_{k}}\right)\right\rangle$. Then, $g^{-} \leq S V L N-I L N W G A\left(g_{1}, g_{2}, \cdots, g_{n}\right) \leq g^{+}$can hold.

(3) Monotonicity: Suppose $g_{k}(k=1,2, \ldots, n)$ is a group of SVLN-ILNs in $L$. If $g_{k} \leq g_{k}^{*}$ for $k=1,2, \ldots, n$, then $S V L N-\operatorname{ILNWGA}\left(g_{1}, g_{2}, \cdots, g_{n}\right) \leq \operatorname{SVLN}-\operatorname{ILNWGA}\left(g_{1}^{*}, g_{2}^{*}, \cdots, g_{n}^{*}\right)$ can hold.

Similar to the proof of Theorem 2, these properties of the SVLN-ILNWGA operator can be also proved, and then the proof of these properties is not repeated here. 


\section{MADM Method Based on the SVLN-ILNWAA or SVLN-ILNWGA Operator}

In the SVLN-ILN setting, we present a MADM method using the SVLN-ILNWAA or SVLN-ILNWGA operator and the score function to handle SVLN-ILN decision making problems corresponding to the pessimistic, moderate, and optimistic attitudes of DMs.

In an SVLN-ILN MADM problem, suppose $G=\left\{G_{1}, G_{2}, \ldots, G_{m}\right\}$ and $Q=\left\{Q_{1}, Q_{2}, \ldots, Q_{n}\right\}$ are represented as a set of alternatives and a set of attributes, respectively. The attribute weigh vector of $Q_{k}(k=1,2, \ldots, n)$ is $\omega=\left(\omega_{1}, \omega_{2}, \ldots, \omega_{n}\right)$ with $\sum_{k=1}^{n} \omega_{k}=1$. Then, the attributes $Q_{k}(k=1,2, \ldots, n)$ over the alternatives $G_{j}(j=1,2, \ldots, m)$ will be evaluated by DMs, which are expressed by SVLN-ILNs from some predefined $\mathrm{LT}$ set $L=\left\{l_{s} \mid s \in[0, z]\right\}$ regarding an even number $z$. In the linguistic evaluation, DM can assign an ILN as the uncertain linguistic argument and an SVLNN as the confident linguistic argument in each SVLN-ILN, so as to give the SVLN-ILN evaluation value of each attribute $Q_{k}(k=1,2, \ldots, n)$ over the alternatives $G_{j}(j=1,2, \ldots, m)$ regarding the LTs. Hence, all SVLN-ILNs can be established as a SVLN-ILN decision matrix $G=\left(g_{j k}\right)_{m \times n}$, where $g_{j k}=\left\langle\left[l_{a_{j k}}, l_{b_{j k}}\right] ; l_{T_{j k}}, l_{I_{j k}}, l_{F_{j k}}\right\rangle$ $(j=1,2, \ldots, m ; k=1,2, \ldots, n)$ is a SVLN-ILN.

Hence, the MADM method using the SVLN-ILNWAA or SVLN-ILNWGA operator and the score function is indicated as the following decision procedure:

Step 1: Compute the aggregated SVLN-ILN $g_{i}=\operatorname{SVLN-ILNWAA}\left(g_{j 1}, g_{j 2}, \ldots, g_{j n}\right)$ or $g_{j}=\operatorname{SVLN}-I L N W G A\left(g_{j 1}, g_{j 2}, \ldots, g_{j n}\right)(j=1,2, \ldots, m)$ based on Equation (9) or Equation (12) for $G_{j}(j=1,2, \ldots, m)$.

Step 2: Calculate the score value of $Y\left(g_{j}\right)$ for each $g_{j}(j=1,2, \ldots, m)$ by Equation (7).

Step 3: Rank the alternatives regarding the score values in a descending order and choose the best one. Step 4: End.

\section{Actual Example and Discussion}

In this section, an actual example is provided to illustrate the applicability of the established MADM method in the SVLN-ILN setting, and then discuss that DMs' attitudes can affect the ranking orders of alternatives and the optimal choice.

\subsection{Actual Example}

In linguistic decision making environment, let us consider that some software company wants to hire a software engineer, which is adapted from the literature [22]. Then, the human resources department preliminarily chooses the four candidates (alternatives) $G_{1}, G_{2}, G_{3}$, and $G_{4}$ from all applicants, and then they require further evaluation by the four attributes: soft skill $\left(Q_{1}\right)$, past experience $\left(Q_{2}\right)$, personality $\left(Q_{3}\right)$, and self-confidence $\left(Q_{4}\right)$. A group of experts or DMs is requested to choose the best candidate by the interview. Then, the weigh vector $\omega=(0.35,0.25,0.2,0.2)$ indicates the importance of the four attributes. Thus, the DMs assess the four possible candidates $G_{j}(j=1,2,3,4)$ over the four attributes $Q_{k}(k=1,2,3,4)$ by SVLN-ILNs from the given LT set $L=\left\{l_{s} \mid s \in[0, z]\right\}$, where $L=\left\{l_{0}\right.$ : extremely poor, $l_{1}$ : very poor, $l_{2}$ : poor, $l_{3}$ : slightly poor, $l_{4}$ : fair, $l_{5}$ : slightly good, $l_{6}$ : good, $l_{7}$ : very good, $l_{8}$ : extremely good $\}$. Thus, all the evaluated SVLN-ILNs can be constructed as the SVLN-ILN decision matrix:

$$
G=\left(g_{j k}\right)_{4 \times 4}=\left[\begin{array}{cccc}
\left\langle\left[l_{4}, l_{6}\right] ; l_{5}, l_{1}, l_{1}\right\rangle & \left\langle\left[l_{4}, l_{6}\right] ; l_{7}, l_{1}, l_{2}\right\rangle & \left\langle\left[l_{4}, l_{7}\right] ; l_{6}, l_{1}, l_{2}\right\rangle & \left\langle\left[l_{5}, l_{6}\right] ; l_{6}, l_{2}, l_{3}\right\rangle \\
\left\langle\left[l_{3}, l_{5}\right] ; l_{7}, l_{2}, l_{3}\right\rangle & \left\langle\left[l_{5}, l_{7}\right] ; l_{6}, l_{3}, l_{5}\right\rangle & \left\langle\left[l_{4}, l_{6}\right] ; l_{4}, l_{1}, l_{1}\right\rangle & \left\langle\left[l_{6}, l_{7}\right] ; l_{6}, l_{1}, l_{2}\right\rangle \\
\left\langle\left[l_{4}, l_{7}\right] ; l_{6}, l_{1}, l_{4}\right\rangle & \left\langle\left[l_{6}, l_{7}\right] ; l_{7}, l_{3}, l_{3}\right\rangle & \left\langle\left[l_{5}, l_{7}\right] ; l_{5}, l_{2}, l_{1}\right\rangle & \left\langle\left[l_{5}, l_{7}\right] ; l_{1}, l_{3}, l_{4}\right\rangle \\
\left\langle\left[l_{6}, l_{7}\right] ; l_{7}, l_{4}, l_{2}\right\rangle & \left\langle\left[l_{5}, l_{7}\right] ; l_{6}, l_{2}, l_{1}\right\rangle & \left\langle\left[l_{4}, l_{6}\right] ; l_{5}, l_{3}, l_{4}\right\rangle & \left\langle\left[l_{5}, l_{6}\right] ; l_{5}, l_{4}, l_{2}\right\rangle
\end{array}\right] .
$$

On the one hand, the established MADM method based on the SVLN-ILNVWAA operator is used for the MADM problem with SVLN-ILN information. Thus, the decision procedure is presented below: 
Step 1: Compute the aggregated value of $g_{1}$ for $G_{1}$ by Equation (9), which is shown as follows:

$$
\begin{aligned}
& g_{1}=S V L N-I L N W A A\left(g_{11}, g_{12}, g_{13}, g_{14}\right)
\end{aligned}
$$

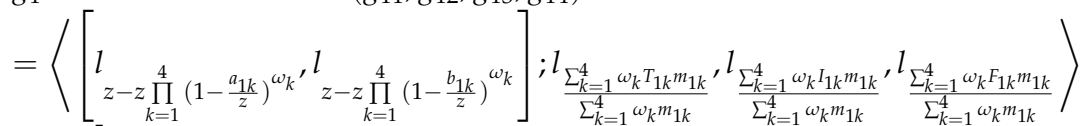

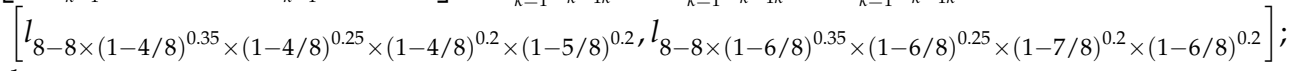

$$
\begin{aligned}
& =\left\langle\begin{array}{l}
\frac{l_{0.35 \times 5 \times(4+6) / 2+0.25 \times 7 \times(4+6) / 2+0.2 \times 6 \times(4+7) / 2+0.2 \times 6 \times(5+6) / 2}}{0.35 \times(4+6) / 2+0.25 \times(4+6) / 2+0.2 \times(4+7) / 2+0.2 \times(5+6) / 2} \\
\frac{l_{0.35 \times 1 \times(4+6) / 2+0.25 \times 1 \times(4+6) / 2+0.2 \times 1 \times(4+7) / 2+0.2 \times 2 \times(5+6) / 2}}{0.35 \times(4+6) / 2+0.25 \times(4+6) / 2+0.2 \times(4+7) / 2+0.2 \times(5+6) / 2}
\end{array},\right. \\
& \frac{l_{0.35 \times 1 \times(4+6) / 2+0.25 \times 2 \times(4+6) / 2+0.2 \times 2 \times(4+7) / 2+0.2 \times 3 \times(5+6) / 2}}{0.35 \times(4+6) / 2+0.25 \times(4+6) / 2+0.2 \times(4+7) / 2+0.2 \times(5+6) / 2} \\
& =\left\langle\left[l_{4.2236}, l_{6.2589}\right] ; l_{5.9038}, l_{1.2115}, l_{1.8750}\right\rangle \text {. }
\end{aligned}
$$

In the similar calculation manner, we can obtain other aggregated values of $g_{j}$ for $G_{j}(j=2,3,4)$ : $g_{2}=\left\langle\left[l_{4.4962}, l_{6.3127}\right], l_{5.8846}, l_{1.8462}, l_{2.9423}\right\rangle, g_{3}=\left\langle\left[\begin{array}{ll}l_{5.002}, & l_{7}\end{array}\right], l_{6.2731}, l_{2.1513}, l_{3.1218}\right\rangle$, and $g_{4}=<\left[l_{5.2427}, l_{6.6805}\right], l_{6.0298}, l_{3.3191}, l_{2.0851}>$.

Step 2: Calculate the score value of $Y\left(g_{1}\right)$ by Equation (7) for $\alpha=0.5$ (considering the moderate attitude of DMs):

$$
\begin{aligned}
& Y\left(g_{1}\right)=\left(\frac{a_{1}+b_{1}}{2 z}+(2 \alpha-1) \frac{b_{1}-a_{1}}{2 z}\right) \times\left(\frac{2 z+T_{1}-I_{1}-F_{1}}{3 z}\right) \\
& =\left(\frac{4.2236+6.2589}{2 \times 8}+(2 \times 0.5-1) \frac{6.2589-4.2236}{2 \times 8}\right) \times\left(\frac{2 \times 8+5.9038-1.2115-1.8750}{3 \times 8}\right) \\
& =0.5137 .
\end{aligned}
$$

In the similar calculation manner, we can obtain other score values of $g_{j}$ for $G_{j}(j=2,3,4)$ : $Y\left(g_{2}\right)=0.4812, Y\left(g_{3}\right)=0.5313$, and $Y\left(g_{4}\right)=0.5162$.

Step 3: Rank the four alternatives as $G_{3} \succ G_{4} \succ G_{1} \succ G_{2}$ based on the score values, and then choose $G_{3}$ as the best candidate among the four candidates.

On the other hand, the established MADM method based on the SVLN-ILNWGA operator is also used for the MADM problem with SVLN-ILN information. Thus, the decision procedure is also presented as follows:

Step 1': Compute the aggregated value of $g_{1}$ for $G_{1}$ by Equation (12):

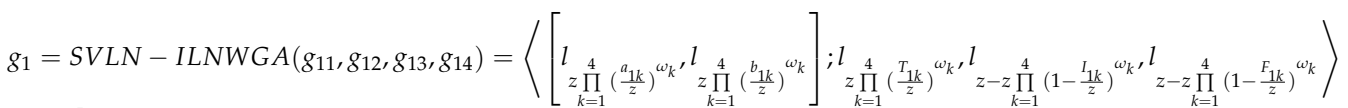

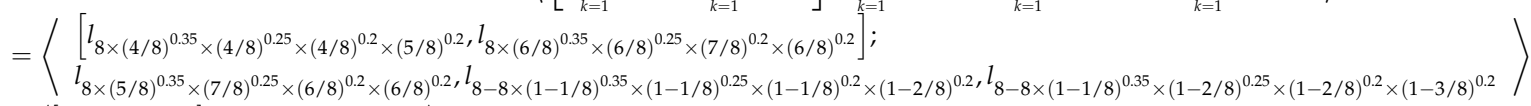

$$
\begin{aligned}
& =\left\langle\left[l_{4.1826}, l_{6.1879}\right] ; l_{5.8503}, l_{1.2125}, l_{1.8941}\right\rangle \text {. }
\end{aligned}
$$

In the similar calculation manner, we can obtain other aggregated values of $g_{j}$ for $G_{j}(j=2,3,4)$ : $g_{2}=<\left[l_{4.1474}, l_{6.0334}\right], l_{5.8393}, l_{1.9027}, l_{3.1183}>, g_{3}=<\left[l_{4.84}, l_{7}\right], l_{6.2007}, l_{2.1662}, l_{3.2696}>$, and $g_{4}=<\left[l_{5.0968}, l_{6.5814}\right]$, $l_{5.8872}, l_{3.3712}, l_{2.25}>$.

Step 2': Calculate the score value of $Y\left(g_{1}\right)$ by Equation (7) for $\alpha=0.5$ (considering the moderate attitude of DMs):

$$
\begin{aligned}
& Y\left(g_{1}\right)=\left(\frac{a_{1}+b_{1}}{2 z}+(2 \alpha-1) \frac{b_{1}-a_{1}}{2 z}\right)\left(\frac{2 z+T_{1}-I_{1}-F_{1}}{3 z}\right) \\
& =\left(\frac{4.1826+6.1879}{2 \times 8}+(2 \times 0.5-1) \frac{6.1879-4.1826}{2 \times 8}\right)\left(\frac{2 \times 8+5.8503-1.2125-1.8941}{3 \times 8}\right) \\
& =0.5062 .
\end{aligned}
$$

In the similar calculation manner, we can obtain other score values of $g_{j}$ for $G_{j}(j=2,3,4)$ : $Y\left(g_{2}\right)=0.4459, Y\left(g_{3}\right)=0.5169$, and $Y\left(g_{4}\right)=0.4947$.

Step 3': Rank the four alternatives as $G_{3} \succ G_{1} \succ G_{4} \succ G_{2}$ based on the score values, and then choose $G_{3}$ as the best candidate among the four candidates. 
Clearly, the best candidate $G_{3}$ is identical although there exists a little difference between two kinds of ranking orders obtained by using the SVLN-ILNWAA and SVLN-ILNWGA operators under the DMs' moderate attitude.

\subsection{Results and Discussion}

Let us consider that the pessimistic, moderate, and optimistic attitudes of DMs may affect their ranking orders. Based on the above similar computational steps, all the decision results based on the SVLN-ILNWAA and SVLN-ILNWGA operators and the DMs' attitudes are shown in Tables 1 and 2.

Table 1. Decision results corresponding to the single-valued linguistic neutrosophic interval linguistic number weighted arithmetic averaging (SVLN-ILNWAA) operator and the decision makers' $\left(\mathrm{DMs}^{\prime}\right)$ attitudes.

\begin{tabular}{ccc}
\hline DMs' Attitude & Score Value & Ranking Order \\
\hline \multirow{2}{*}{ Pessimist $(\alpha=0)$} & $Y\left(g_{1}\right)=0.4139, Y\left(g_{2}\right)=0.4004$, & $G_{4} \succ G_{3} \succ G_{1} \succ G_{2}$ \\
& $Y\left(g_{3}\right)=0.4429, Y\left(g_{4}\right)=0.4540$ & \\
Moderate $(\alpha=0.5)$ & $Y\left(g_{1}\right)=0.5137, Y\left(g_{2}\right)=0.4812$, & $G_{3} \succ G_{4} \succ G_{1} \succ G_{2}$ \\
& $Y\left(g_{3}\right)=0.5313, Y\left(g_{4}\right)=0.5162$ & \\
Optimist $(\alpha=1)$ & $Y\left(g_{1}\right)=0.6134, Y\left(g_{2}\right)=0.5621$, & $G_{3} \succ G_{1} \succ G_{4} \succ G_{2}$ \\
\hline
\end{tabular}

Table 2. Decision results corresponding to the SVLN-ILN weighted geometric averaging (SVLN-ILNWGA) operator and the DMs' attitudes.

\begin{tabular}{|c|c|c|}
\hline DMs' Attitude & Score Value & Ranking Order \\
\hline Pessimist $(\alpha=0)$ & $\begin{array}{l}Y\left(g_{1}\right)=0.4083, Y\left(g_{2}\right)=0.3633 \\
Y\left(g_{3}\right)=0.4226, Y\left(g_{4}\right)=0.4318\end{array}$ & $G_{4} \succ G_{3} \succ G_{1} \succ G_{2}$ \\
\hline Moderate $(\alpha=0.5)$ & $\begin{array}{l}Y\left(g_{1}\right)=0.5062, Y\left(g_{2}\right)=0.4459 \\
Y\left(g_{3}\right)=0.5169, Y\left(g_{4}\right)=0.4947\end{array}$ & $G_{3} \succ G_{1} \succ G_{4} \succ G_{2}$ \\
\hline Optimist $(\alpha=1)$ & $\begin{array}{l}Y\left(g_{1}\right)=0.6041, Y\left(g_{2}\right)=0.5285 \\
Y\left(g_{3}\right)=0.6112, Y\left(g_{4}\right)=0.5576\end{array}$ & $G_{3} \succ G_{1} \succ G_{4} \succ G_{2}$ \\
\hline
\end{tabular}

Obviously, two kinds of ranking orders based on the SVLN-ILNWGA and SVLN-ILNWGA operators in Tables 1 and 2 are identical under the DMs' pessimistic or optimistic attitudes, but the DMs' attitudes can affect the ranking orders. Then, the best candidate is $G_{4}$ for pessimist or $G_{3}$ for optimist and the worst one is $G_{2}$ in all ranking orders. Hence, the established SVLN-ILN MADM method shows its sensitivity and flexibility regarding the DMs' attitudes, which depend on their preference.

As the decision information in this study uses the SVLN-ILN that is composed of ILN (uncertain/interval linguistic argument part) and SVLNN (confident linguistic argument part) for the first time, the SVLN-ILN MADM method is established for the first time because there is no other study in existing literature. Therefore, existing various linguistic MADM methods cannot carry out such a decision making problem with SVLN-ILN information in this paper.

Generally, this study indicates a new concept of SVLN-ILN and a new SVLN-ILN MADM method, and then DMs can choose one of the SVLN-ILNWAA and the SVLN-ILNWGA operators to apply the established MADM method to MADM problems with SVLN-ILN information and their preference attitude or actual requirements.

\section{Conclusions}

This study proposed the SVLN-ILN concept to express the hybrid information of both a single-valued LNN and an ILN, the operational laws of SVLN-ILNs, and the score function of SVLN-ILN, along with the attitude index and confident degree for ranking SVLN-ILNs. Then, the SVLN-ILNWAA and SVLN-ILNWGA operators were presented in order to aggregate SVLN-ILN 
information, and then their advantage is that all the LT values in their aggregated SVLN-ILN can still belong to the predefined LT set, rather than beyond the LT set in some linguistic operations [8,9]. It is well known that the two weighted aggregation operators are not only the most basic and simplest operations, but also two main mathematical tools in MADM problems. Hence, an MADM method was established based on the SVLN-ILNWAA or SVLN-ILNWGA operator and the score function so as to handle MADM problems with SVLN-ILN information and DMs' attitudes, which existing MADM methods cannot handle. By an actual example, the decision results illustrated the applicability of the established MADM method in the SVLN-ILN setting.

This study proposed for the first time the expression and score problems of hybrid information of both the SVLN number and ILN using the SVLN-ILN and the weighted aggregation problems of SVLN-ILNs to realize MADM problems with both interval/uncertain linguistic arguments (linguistic uncertainty) and linguistic neutrosophic arguments (confident level/degree). Then, the established SVLN-ILN MADM method contains much more linguistic information (interval/uncertain linguistic arguments and confident linguistic arguments) and indicates its flexibility for DMs' preference attitudes along with pessimist, moderate, and optimist in linguistic the decision making process, which are the main advantages in this study. From the viewpoint of scientific potential impact, the proposed technologies will be extended to medical diagnosis, hospital service quality evaluation, selection of suppliers, machining process selection, and so on.

Author Contributions: J.Y. proposed the SVLN-ILN concept, the SVLN-ILNWAA and SVLN-ILNWGA operators, the score function, and the MADM method. W.C. provided the calculation of examples and comparative analysis. All authors wrote the paper together.

Funding: This research received no external funding.

Acknowledgments: This study was supported by the National Natural Science Foundation of China (Nos. 71471172, 61703280).

Conflicts of Interest: The authors declare no conflict of interest.

\section{References}

1. Chatterjee, P.; Mondal, S.; Boral, S.; Banerjee, A.; Chakraborty, S. A novel hybrid method for non-traditional machining process selection using factor relationship and multi-attributive border approximation method. Facta Univ. Ser. Mech. Eng. 2017, 15, 439-456. [CrossRef]

2. Petković, D.; Madić, M.; Radovanović, M.; Gečevska, V. Application of the performance selection index method for solving machining modm problems. Facta Univ. Ser. Mech. Eng. 2017, 15, 97-106. [CrossRef]

3. Roy, J.; Adhikary, K.; Kar, S.; Pamučar, D. A rough strength relational DEMATEL model for analysing the key success factors of hospital service quality. Decis. Mak. Appl. Manag. Eng. 2018, 1, 121-142. [CrossRef]

4. Vasiljevic, M.; Fazlollahtabar, H.; Stević, Ž.; Vesković, S. A rough multicriteria approach for evaluation of the supplier criteria in automotive industry. Decis. Mak. Appl. Manag. Eng. 2018, 1, 82-96. [CrossRef]

5. Zadeh, L.A. The concept of a linguistic variable and its application to approximate reasoning Part I. Inf. Sci. 1975, 8, 199-249. [CrossRef]

6. Herrera, F.; Herrera-Viedma, E.; Verdegay, L. A model of consensus in group decision making under linguistic assessments. Fuzzy Sets Syst. 1996, 79, 73-87. [CrossRef]

7. Herrera, F.; Herrera-Viedma, E. Linguistic decision analysis: Steps for solving decision problems under linguistic information. Fuzzy Sets Syst. 2000, 115, 67-82. [CrossRef]

8. $\mathrm{Xu}, \mathrm{Z}$.S. A method based on linguistic aggregation operators for group decision making with linguistic preference relations. Inf. Sci. 2004, 166, 19-30. [CrossRef]

9. $\mathrm{Xu}, \mathrm{Z} . \mathrm{S}$. A note on linguistic hybrid arithmetic averaging operator in multiple attribute group decision making with linguistic information. Group Decis. Negot. 2006, 15, 593-604. [CrossRef]

10. Merigó, J.M.; Casanovas, M.; Martínez, L. Linguistic aggregation operators for linguistic decision making based on the Dempster-Shafer theory of evidence. Int. J. Uncertain. Fuzziness Knowl. Based Syst. 2010, 18, 287-304. [CrossRef] 
11. Xu, Y.J.; Merigó, J.M.; Wang, H.M. Linguistic power aggregation operators and their application to multiple attribute group decision making. Appl. Math. Model. 2012, 36, 5427-5444. [CrossRef]

12. Merigó, J.M.; Casanovas, M.; Palacios-Marqués, D. Linguistic group decision making with induced aggregation operators and probabilistic information. Appl. Soft Comput. 2014, 24, 669-678. [CrossRef]

13. Merigó, J.M.; Palacios-Marqués, D.; Zeng, S.Z. Subjective and objective information in linguistic multi-criteria group decision making. Eur. J. Oper. Res. 2016, 248, 522-531. [CrossRef]

14. Yu, D.J.; Li, D.F.; Merigó, J.M.; Fang, L.C. Mapping development of linguistic decision making studies. J. Intell. Fuzzy Syst. 2016, 30, 2727-2736. [CrossRef]

15. Xu, Z.S. Uncertain linguistic aggregation operators based approach to multiple attribute group decision making under uncertain linguistic environment. Inf. Sci. 2004, 168, 171-184. [CrossRef]

16. Xu, Z.S. Induced uncertain linguistic OWA operators applied to group decision making. Inf. Fusion 2006, 7, 231-238. [CrossRef]

17. Wei, G.W. Uncertain linguistic hybrid geometric mean operator and its application to group decision making under uncertain linguistic environment. Int. J. Uncertain. Fuzziness Knowl. Based Syst. 2009, 17, 251-267. [CrossRef]

18. Park, J.H.; Gwak, M.G.; Kwun, Y.C. Uncertain linguistic harmonic mean operators and their applications to multiple attribute group decision making. Computing 2011, 93, 47. [CrossRef]

19. Wei, G.W.; Zhao, X.F.; Lin, R.; Wang, H.J. Uncertain linguistic Bonferroni mean operators and their application to multiple attribute decision making. Appl. Math. Model. 2013, 37, 5277-5285. [CrossRef]

20. Zhang, H. Uncertain linguistic power geometric operators and their use in multiattribute group decision making. Math. Probl. Eng. 2015, 2015, 948380. [CrossRef]

21. Ye, J. Aggregation operators of neutrosophic linguistic numbers for multiple attribute group decision making. SpringerPlus 2016, 5, 1691. [CrossRef] [PubMed]

22. Ye, J. Multiple attribute decision-making method based on linguistic cubic variables. J. Intell. Fuzzy Syst. 2018, 34, 2351-2361. [CrossRef]

23. Fang, Z.B.; Ye, J. Multiple attribute group decision-making method based on linguistic neutrosophic numbers. Symmetry 2017, 9, 111. [CrossRef]

24. Wang, J.Q.; Han, Z.Q.; Zhang, H.Y. Multi-criteria group decision-making method based on intuitionistic interval fuzzy information. Group Decis. Negot. 2014, 23, 715-733. [CrossRef]

25. Wan, S.P. Method of attitude index for interval multi-attribute decision-making. Control Decis. 2009, 24, 35-38. 